\title{
DECOMPOSING, RECOMPOSING, AND SITUATING CIRCADIAN MECHANISMS: THREE TASKS IN DEVELOPING MECHANISTIC EXPLANATIONS
}

\author{
William BeCHTEL and AdELE ABRAHAMSEN \\ University of California, San Diego
}

Reductionist inquiry, which involves decomposing a mechanism into its parts and operations, is only one of the tasks of mechanistic research. A second task (which may be undertaken largely simultaneously) is recomposing it - conceptually reassembling the parts and operations into an organized arrangement that constitutes the mechanism. Other tasks include determining how multiple operations are orchestrated in real time, and investigating how the mechanism interacts with the environment in which it is situated.

Accordingly, explaining how a mechanism generates a phenomenon requires integrating research from at least two levels of organization - one involving the parts and their operations and another involving the mechanism as a whole. Sometimes additional levels are so salient that they too must be incorporated into the account. Researchers may come to recognize, for example, that the target mechanism is itself part of a larger integrated system (a higher-level mechanism) that confronts its own environment, and that operations within the target mechanism may be affected as the larger system confronts varying environmental conditions. Choosing mechanistic explanation as the preferred framework for pursuing questions of reduction both illuminates the importance of reductionistic inquiry and reveals that it must be complemented by other kinds of explanatory work.

Although the modern pursuit of mechanistic explanation has a history spanning several centuries in the life sciences, it is only recently that certain philosophers focused on biology have subjected this pursuit to systematic analysis. The new mechanistic philosophy of science that has emerged from that endeavor remains unfamiliar to many other philosophers. Accordingly, we begin with a brief account of mechanistic explana- 
tion and the roles of decomposition and recomposition in developing such explanations. We then illustrate these two tasks of mechanistic research by examining their interplay in research on circadian rhythms. After briefly introducing the phenomena of circadian rhythms, we present highlights of the attempts to decompose the responsible mechanisms - research that began in the 1970s and has advanced rapidly since the early 1990s. We then address how the very success of this research has prompted new inquiries into how the parts and operations are integrated so as to constitute a mechanism and how those operations are orchestrated. Finally, we add another level of analysis by considering how that mechanism is situated in and affected by a higher-level mechanism.

The account we offer here stands in sharp contrast to a common philosophical characterization of reduction according to which the reducing science (e.g., molecular biology) explains all of the phenomena originally explained by the reduced science (e.g., neurophysiology), which thereby loses its autonomy (Churchland, 1986; Bickle, 2003). On that view, the reduced science at best provides shorthand accounts for the more complete accounts offered in the reducing science. Accordingly, a primary line of argument for opponents of reduction (e.g., Fodor, 1974) has been that the reducing science cannot account for certain important regularities (laws) in the sciences to be reduced. Since these regularities therefore remain in the province of an autonomous, unreduced science, the attempt at reduction is said to have failed. It is important to note that this characterization of reduction and the counterarguments against it are tied specifically to the deductive-nomological (D-N) account of explanation. As characterized by Hempel (1965), explanation involves deriving statements describing phenomena to be explained from statements of laws and initial conditions. In the hands of philosophers of science such as Nagel (1961), this led to the theory-reduction account of science, according to which reduction explains the laws in the earlier (reduced) theory by deriving them from the laws of a more basic science (the reducing theory) together with bridge principles and boundary conditions (for historical review and critical discussion, see Bechtel \& Hamilton, 2007). This account is especially problematic when applied to the life sciences, where explanations invoke laws infrequently (if laws are even available), but appeal to mechanisms regularly. Mechanistic explanations, as we will see, require integrating processes at different levels and do not invite supplanting accounts at one level with those from a different level. 
A mechanistic explanation starts with a phenomenon produced by some system across a certain range of conditions. The phenomenon is explained by construing the system as a mechanism and describing how that mechanism works. In a previous paper, we characterized a mechanism as "a structure performing a function in virtue of its component parts, component operations, and their organization" (Bechtel \& Abrahamsen, 2005; for related accounts, see Bechtel \& Richardson, 1993; Glennan, 2002; Machamer, Darden, \& Craver, 2000). In order to develop a mechanistic explanation it is necessary to decompose the mechanism into parts and/or the operations they perform. Doing so often requires specialized instruments and techniques since naturally functioning systems typically conceal their parts and operations. Many of the advances in modern biology have stemmed from the development of particularly effective means for decomposing particular classes of biological systems. Scientists have not explained a phenomenon, however, until they have recomposed the mechanism - determined how it is organized and interacts with its environment. As challenging as is decomposition, recomposition often is even more difficult. Parts and operations are often highly integrated with one another so that as one part of the mechanism changes, numerous other parts are affected and change their operations. If the organization involves cycles, the effects of a part's operations may feed back (positively or negatively) on it, affecting operations on the next cycle. Ambitious modeling projects, sometimes using the available tools for analyzing dynamical systems, often are required to understand the consequences of organization and particularly the orchestration of multiple operations in real time (Bechtel \& Abrahamsen, in press).

Many scientists regard the process of decomposition as reductionistic, but it is important to recognize how their conception of reduction differs from the philosophical conception described above. The goal of decomposition is to determine what parts and operations together make a functioning mechanism. The operations performed by the parts are quite unlike the activity of the whole mechanism. (For example, the valves in a heart open and close, but the heart itself pumps blood.) The components must be appropriately organized and the mechanism situated in an appropriate environment to realize the phenomenon (valves and chambers of the heart have a proper spatial organization, their operations have proper temporal organization, and the heart must be properly localized, supported, and 
connected to veins and arteries). To figure out the organization within the mechanism, scientists must recompose what they have decomposed. We will illustrate these two complementary tasks by examining their interplay in research on circadian rhythms. First, though, we will delineate the phenomena to be explained.

\section{Delineating Circadian Phenomena}

The ability of organisms to keep track of the time of day and respond appropriately to it, even when deprived of external cues such as exposure to sunlight, has fascinated investigators since ancient times. (Androsthenes of Thasus, a captain in Alexander's fleet, recorded the daily movement of the leaves of the tamarind tree, while Hippocrates and Galen both observed how body temperature in patients with fevers varied with time of day). Subsequently, circadian rhythms have been found in a wide variety of living organisms, from cyanobacteria to plants, fungi, and a variety of animals including Drosophila, mice, and humans. They affect biochemical processes (e.g. protein synthesis), physiological functions (e.g., digestion), behavioral phenomena (e.g., locomotor activity), and cognitive performance (e.g., reaction times). Systematic study of these rhythms - incorporating experimental methods in addition to finer-grained description - began only in the middle of the $20^{\text {th }}$ century and yielded a richer characterization of the phenomena. In particular, early experiments maintaining organisms in regulated conditions (constant darkness, or light exposure limited to certain hours) demonstrated that these rhythms (1) are endogenously controlled, (2) are entrainable by Zeitgebers (environmental cues such as onset of daylight or temperature changes), and (3) are temperature compensated (the period of oscillation remains nearly the same across a wide range of temperatures). Halberg (1959) termed these rhythms circadian (circa = about + dies $=$ day) because when Zeitgebers are removed, the periods are regular but with periods varying slightly from 24 hours.

\section{Decomposing AND Recomposing THE CiRCADIAN System}

We will primarily focus on the quest to explain how animals maintain rhythms endogenously. Investigations in the 1970s, some focusing on mammals and some on fruit flies (Drosophila), began to provide clues as 
to the mechanisms underlying circadian rhythms. First, mammalian researchers traced the maintenance of these rhythms to a specific region of the brain - the suprachiasmatic nucleus $(\mathrm{SCN})$ - which is regarded as the central clock. ${ }^{1}$ The SCN is a bilateral structure located just above the optic chiasm, where projections from the two eyes come together, with each side comprising approximately 8,000 to 10,000 neurons. Evidence that the SCN serves as a central clock came from demonstrations that (1) it receives appropriate projections from the eyes for entrainment by light; (2) damage to it eliminates endogenous rhythms; and (3) it can sustain its own rhythms (Inouye \& Kawamura, 1979).

The mammalian research identified the locus of the central clock, but not how it worked. The task of decomposing the mechanism into its component parts and operations was begun by going inside individual Drosophila neurons to determine how they sustained oscillations. The first breakthrough was Konopka and Benzer's (1971) identification of a specific gene - period (per) - in which mutations yielded arrhythmic flies or ones with significantly shortened or lengthened rhythms. Rosbash and his colleagues cloned per in the $1980 \mathrm{~s}$, enabling them to determine that concentrations of both per mRNA and PER (the protein synthesized from per) exhibited circadian oscillations (Hardin, Hall, \& Rosbash, 1990). At this point they could begin to coordinate decomposition with recomposition (figuring out spatial and temporal organization of the parts and operations). One clue was that peaks and valleys in concentrations of PER lagged behind those of per mRNA by approximately 8 hours. Moreover, since PER was detected not only in cytoplasm (where it is synthesized) but also in the nucleus, they proposed a mechanism incorporating a negative feedback loop to explain circadian oscillations in Drosophila. Its parts, operations, and organization are illustrated in Figure 1. On this account, synthesis of the protein PER is initiated by the transcription of per into per mRNA, which must be transported to the cytoplasm to be translated into PER. Several hours thereafter PER is transported back into the nucleus, where it slows synthesis of additional PER by somehow inhibiting per transcription. (Though not shown, per eventually is released from inhibition by the breakdown of nuclear PER, allowing a new turn of the 24-hour cycle to begin.)

\footnotetext{
${ }^{1}$ The determination that a specific set of neurons in Drosophila, the lateral neurons, comprised its central clock was not made until much later (Helfrich-Förster, 1996).
} 


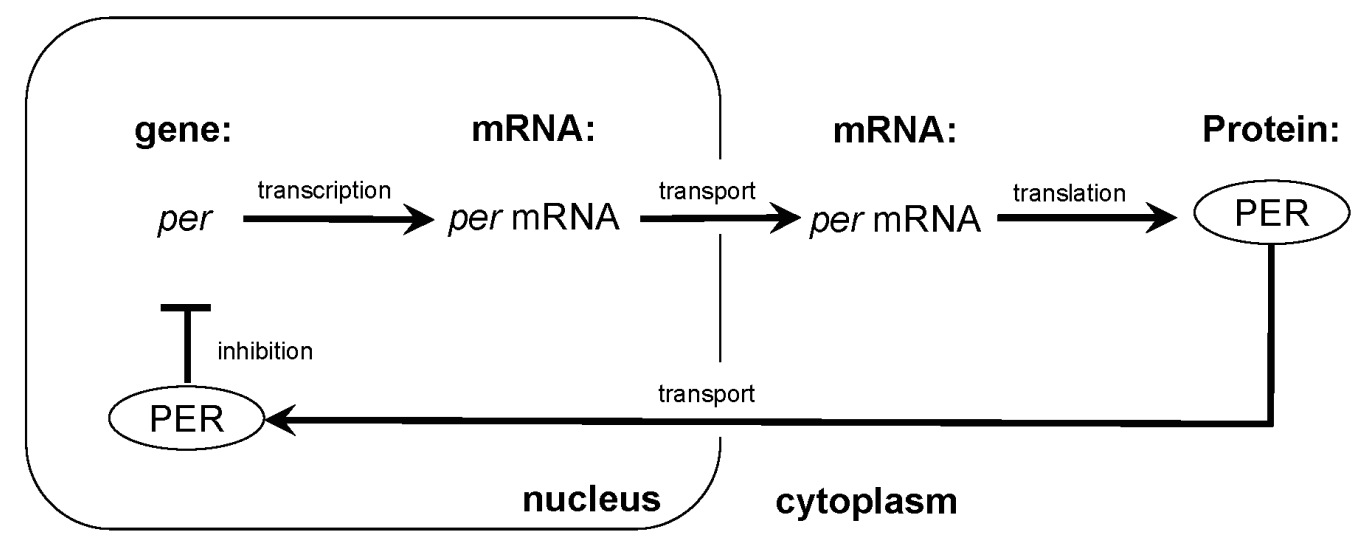

Figure 1: Proposed mechanism for generating circadian rhythms in Drosophila (Hardin, Hall, \& Rosbash, 1990).

Hardin et al.'s proposal generated a host of additional questions. Why do concentrations of PER lag behind those of per mRNA? What determines when it is transported into the nucleus? Precisely how does PER inhibit per transcription? Over the next 15 years, research addressing these questions brought discovery of a host of additional genes and proteins and determination of how they are involved in the feedback loop whereby PER inhibits per. For example, it was found that after PER is synthesized it forms a compound with another protein, TIMELESS (TIM), and that only as part of the compound does it enter the nucleus. Moreover, the ability of the PER:TIM compound to inhibit per transcription involves it acting on another compound formed from a third protein (CLOCK) and fourth protein (CYCLE). This interaction inhibits the ability of CLOCK:CYCLE to bind to the promoter site on per and tim DNA and hence to activate their transcription. Entrainment was found to involve an additional protein, CRYPTOCHROME (CRY), which in response to light serves to degrade TIM, and thus to release CLOCK:CYCLE from inhibition by PER:TIM (for details, see Bechtel, in press-b). Figure 2 shows how these components fit into the mechanism sketched in Figure 1. As a result of these and other discoveries, the number of components and the range of operations in the clock increased dramatically. 


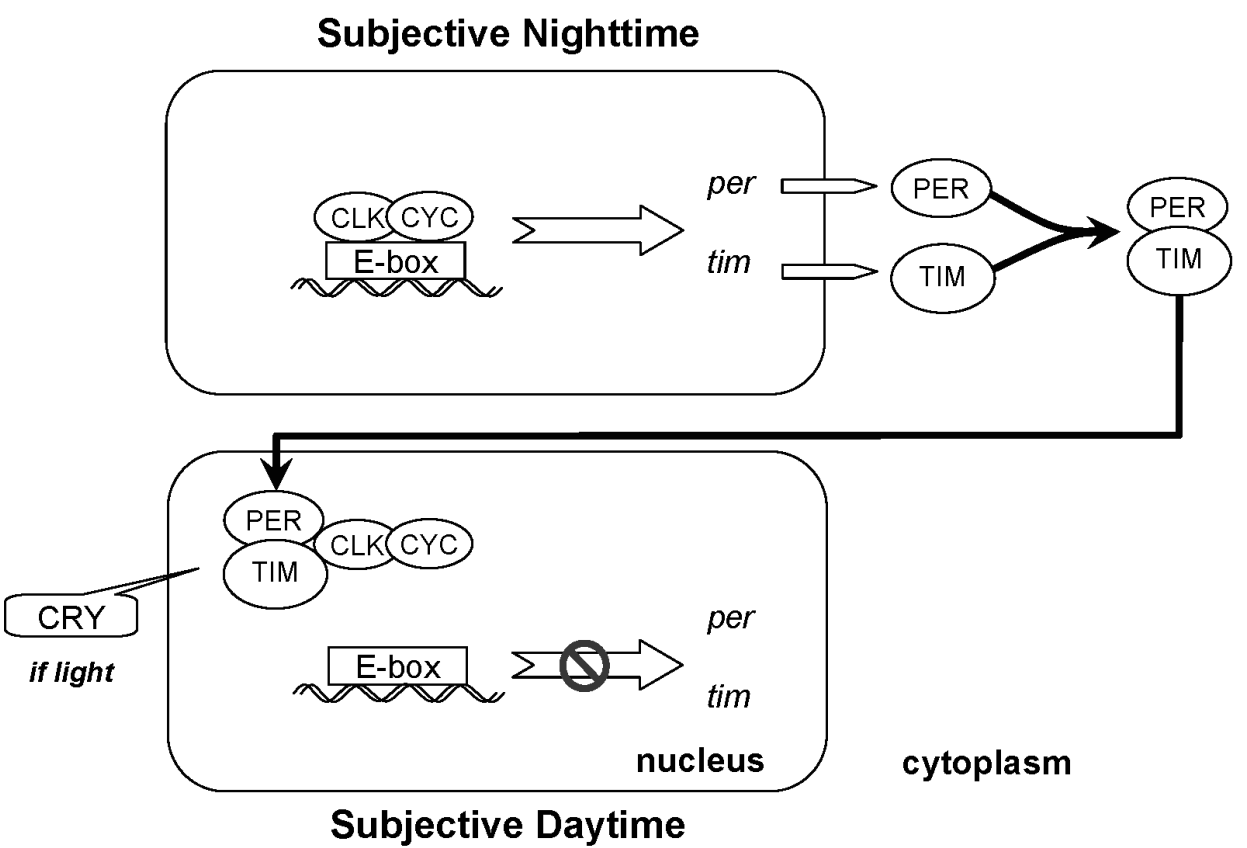

Figure 2. A fuller account of the feedback mechanism that maintains circadian rhythms in Drosophila. It includes component parts and operations that were discovered in the late 1990s and shows how the mechanism's dynamics differ between nighttime and daytime. The large open arrow indicates whether or not gene transcription is activated. The smaller open arrows represent the operations of gene expression (transcription, transport, and translation) that were separately indicated in Figure 1.

Most of these advances stemmed directly from research on Drosophila, but the discovery of CLOCK actually resulted from mammalian researchers seeking mutations that affected mammalian circadian rhythms. A homolog of the mammalian gene was then found in Drosophila. Subsequently, homologues of several Drosophila genes were found in mammals. The circadian clock, like many biological mechanisms, is largely conserved through phylogeny. Phylogenetic conservation is not perfect, however; often key components are retained but significantly modified. CRY, for example, subserves entrainment in Drosophila. In mammals it no longer performs that role, but replaces TIM in forming a compound with PER. This discovery prompted a search for a protein that could replace CRY in subserving entrainment in mammals, culminating in the identification of melanopsin. But the way in which these molecules subserve entrainment was found to differ: melanopsin (in mammals) promotes per transcription, whereas CRY (in Drosophila) indirectly releases it from inhibition by degrading TIM. The assumption of conservation thus served as a discovery 
heuristic both when direct homologues of genes in one order of animals were found in another and when differences were uncovered (Bechtel, in press-c). While additional parts and operations undoubtedly remain to be identified, these efforts to decompose the circadian clock have yielded an extensive catalog of clock parts and operations in both Drosophila and mammals and confidence that the basic component parts and operations in the clock are now known.

\section{OrChestrating AND SituATING THE CiRCADIAN System}

The scientists discussed successfully executed their task of decomposition, identifying a number of genes and proteins in the circadian clock and the operations they perform. They were similarly successful in their complementary task of recomposition, offering a well-supported account of how these component parts and operations were organized. A different group of scientists could now use computational modeling to explore how the operations were orchestrated so as to produce sustained periodic oscillations. In addition to the negative feedback loop shown in Figure 1 through which PER serves to inhibit its own transcription, there is a positive feedback loop involved in the generation of CLOCK. The combination of positive and negative feedback loops and the nonlinear nature of the various reactions render it impossible to determine simply through mental simulations whether periodic oscillations will result and can be sustained rather than dampen. Using differential equations for quantifying and relating the various operations, Goldbetter (1995; Leloup \& Goldbeter, 1998) developed a model that showed that with biologically plausible values for key parameters, such a mechanism would exhibit sustained periodic oscillations. (For details, and discussion of other contributions of computational modeling to understanding the orchestration of circadian mechanisms, see Bechtel, in press-a.)

The importance of situating the intracellular mechanisms in a larger context was recognized when Welsh, Logothetis, Meister, and Reppert (1995) found that individual SCN neurons dispersed in culture exhibited a large range of variation (21.25 to 26.25 hours) and standard deviation (1.2 hours) in their oscillations. This finding indicated that the close to 24-hour rhythms exhibited in the circadian controlled activities in whole organisms depends on synchronization between SCN cells. Although several mechanisms have been considered, evidence points to synchronization being 
achieved via the release and uptake of hormones such as vasoactive intestinal polypeptide (Aton, Colwell, Harmar, Waschek, \& Herzog, 2005). Such synchronization involves individual oscillators modifying their behavior as a result of the signals they receive from other oscillators to which they are coupled. As in the case of relating the operations of parts within cells, researchers turned to computational modeling to understand conditions under which synchronization can be achieved.

Other research points to the need to situate the entire central clock (SCN in mammals) within a yet larger system. In order to be entrained by Zeitgebers (via the mechanism involving genes and proteins within neurons as described above), the SCN must have inputs from sensory organs. Moreover, the SCN must send signals to other (peripheral) organs of the body if it is to affect their activity. In the early stages of research, investigators assumed a linear arrangement of signals from sensory organs to the central clock and from the central clock to peripheral organs. This assumed arrangement, however, has been severely challenged as a result of further investigation. There is evidence that photoreceptive proteins are themselves modified by the SCN, so that the SCN is modulating its own entrainment system (Roenneberg, Daan, \& Merrow, 2003). Further, already in the 1990s it became apparent that cells in the output organs contain the same clock genes and proteins as the SCN and that cells in peripheral organs of the body exhibit oscillations in clock proteins when they receive input from the SCN. Since these oscillations appeared to dampen when peripheral cells did not receive SCN inputs, the peripheral oscillators initially were viewed as slaves. However, there are now findings that suggest the apparent dampening of peripheral oscillators may be due to desynchronization, not to cessation of oscillation (Welsh, Yoo, Liu, Takahashi, \& Kay, 2004). Moreover, some of the peripheral oscillators have their own Zeitgebers (e.g., liver oscillators respond to the period of feeding). They also seem capable themselves of altering oscillation within the SCN (Panda \& Hogenesch, 2004). Increasingly, as researchers situate the central clock (SCN) mechanism in a larger system responsible for circadian oscillations, they realize that there are extensive feedback connections rendering it a highly integrated system, as illustrated in Figure 3. 


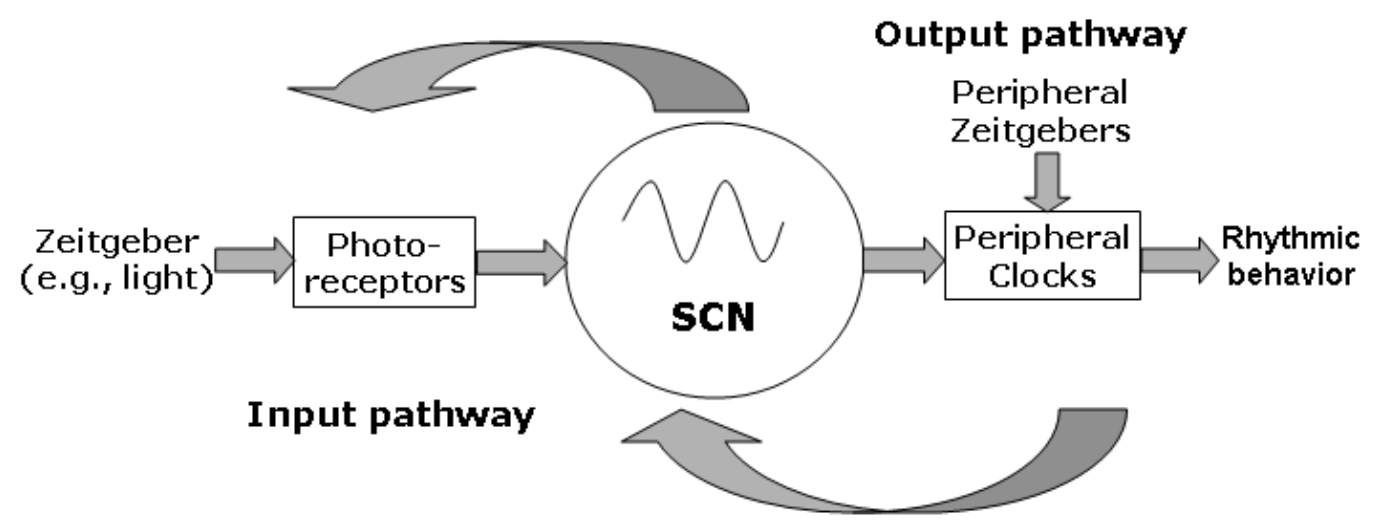

Figure 3. Feedback (curved arrows) help integrate components of the overall system responsible for circadian rhythms. Previously these components were construed as forming a linear system (straight arrows).

Although it was possible to investigate experimentally how individual SCN neurons functioned when isolated from other components, and this provided enormous insight into the parts and operations within them, a full understanding of how circadian behavior is produced requires conceptually recomposing the whole mechanism within these neurons and situating it in relation to other SCN neurons and also other organs of the body. Studies of individual SCN neurons in isolation resulted only in a first approximation - in recomposing and situating the mechanism, researchers must modify their initial accounts to incorporate what is learned about how component parts and operations are, in part, altered by activities occurring in the broader environment.

\section{CAusation at MultiPle LeVEls Without Elimination OR INTERLEVEL CAUSATION}

The account of research on circadian rhythms we have presented supports a very different perspective on reduction than that commonly encountered in philosophy. Reductionist inquiry involved decomposing the central clock into component genes and proteins within each of its neurons. To understand how these components constituted a clock, however, investigators had to recompose the mechanism - ascertain that these components were organized into a feedback loop in which a clock gene, per, is expressed in a protein which then serves to inhibit its own expression. Further pursuit of this inquiry identified a host of additional genes and proteins. The discov- 
ery of these parts and their operations did not end the explanatory endeavor, however, as recomposition was required to understand their contribution to the overall mechanism. Moreover, the individual neurons are constituents of a larger system (the $\mathrm{SCN}$ ), and the way they are coordinated within that system affects their individual behavior. As well, that system functions as part of a yet larger integrated system involving sensory inputs and peripheral oscillators, and these affect the behavior of genes and proteins within the central clock mechanism. The accounts of these higherlevel systems were not obtained by deriving them from what is known of the genes and proteins within individual cells. Rather, they had to be discovered (and are still being discovered) by using investigatory tools appropriate for the level at which the entities of current interest are interacting. There is no possibility of eliminating the account of these higher-level processes in favor of accounts of lower-level processes, since what is learned at the lower level does not provide a complete account of the phenomenon.

When speaking of decomposing a mechanism into its parts or recomposing the whole, it is natural to speak, as we have, of the parts residing at a lower level than the mechanism as a whole (and the mechanism as at a lower level than any system of which it is a constituent). This introduction of levels into the account of mechanisms, however, is highly circumcised as it is dependent on the decomposition of particular mechanisms. The level down from the mechanism as a whole consists of those parts whose operations contribute to the functioning of that mechanism. They are not necessarily of the same size - they are related only insofar as their operations are coordinated to produce the phenomenon of interest. Moreover, if researchers take any two components of a mechanism apart, there is no basis for determining whether these yet lower-level components all reside at one common level (Craver, 2007).

In accounting for the behavior of a mechanism, reductionist researchers seek to determine how the parts interact with each other causally. When parts perform their operations, the mechanism as a whole is affected, and this can result in the mechanism functioning differently in its environment. Also, the causal engagement of the mechanism with entities in its environment affects the way its parts operate. The notions of bottom-up and top-down causation are sometimes invoked to characterize these relations, but Craver and Bechtel (2007) have argued against extending causation to the interlevel context on grounds that this leads to unnecessary conceptual problems. For example, causes usually precede their effects, but 
something happening at one level does not precede the corresponding change at another level - when a part of a mechanism is altered, the mechanism as a whole is altered simultaneously, and vice versa. Instead of extending the notion of causation to interlevel contexts, Craver and Bechtel advocated characterizing interlevel relations in terms of constitution (i.e., part-whole relations in the mechanism). They emphasized that any changes to the whole mechanism are also changes to at least one of its parts, and changes to a part are also changes to the whole. If the change to the part is brought about by interaction with other parts, then that is the level at which causation is identified. If, on the other hand, the mechanism as a whole is changed by interactions with other mechanisms, then the causal relation is between those mechanisms. By combining intralevel causal analysis at multiple levels with interlevel constitution relations, one can account for the phenomena for which others invoke the notions of bottom-up and topdown causation without unnecessary conceptual problems.

The idea that reductionistic research can eliminate the need for higher-level accounts stems in part from the assumption that there is a lowest level at which all causal processes can be described. The account of levels just sketched does not require us to embrace the idea of a lowest level of entities out of which all higher-level entities are built. And even more emphatically, it does not support the idea that a comprehensive causal account can be provided at the lowest level. Rather, the mechanistic project starts with identifying a phenomenon that one wants to explain. Researchers posit that the phenomenon is due to a mechanism behaving in a certain way under specified conditions. Successful mechanistic inquiry reveals how it is that the mechanism is able to exhibit the behavior under those conditions (by identifying the parts, operations, and organization, and orchestration within a mechanism), but it does not itself explain how those conditions arise. In the course of mechanistic inquiry researchers may discover other conditions that impact the mechanism and affect whether it exhibits the behavior. These causal relations are not explained by the account identifying the parts and operations within the mechanism and their organization - they are viewed as additional factors that are included in the full mechanistic explanation of the phenomenon. The reductionist (decompositional) task of mechanistic research provides part, but only part, of the overall endeavor of mechanistic explanation. 


\section{REFERENCES}

Aton, S.J., Colwell, C.S., Harmar, A.J., Waschek, J., \& Herzog, E.D. (2005). Vasoactive Intestinal Polypeptide Mediates Circadian Rhythmicity and Synchrony in Mammalian Clock Neurons. Nature Neuroscience, 8, pp.476-483.

Bechtel, W. (in press-a). Computational Modeling and Mechanistic Explanation: Understanding Circadian Rhythms. Studies in History and Philosophy of Science Part A.

Bechtel, W. (in press-b). The Downs and Ups of Mechanistic Research: Circadian Rhythm Research as an Exemplar. Erkenntnis.

Bechtel, W. (in press-c). Generalization and Discovery through Conserved Mechanisms: Cross Species Research on Circadian Oscillators. Philosophy of Science.

Bechtel, W., \& Abrahamsen, A. (2005). Explanation: A Mechanist Alternative. Studies in History and Philosophy of Biological and Biomedical Sciences, 36, pp.421441.

Bechtel, W., \& Abrahamsen, A. (in press). Complex Biological Mechanisms: Cyclic, Oscillatory, and Autonomous. In C.A. Hooker (Ed.), Philosophy of Complex Systems. Handbook of the Philosophy of Science, Volume 10. New York: Elsevier.

Bechtel, W., \& Hamilton, A. (2007). Reduction, Integration, and the Unity of Science: Natural, Behavioral, and Social Sciences and the Humanities. In T. Kuipers (Ed.), Philosophy of Science: Focal Issues. New York: Elsevier.

Bechtel, W., \& Richardson, R. C. (1993). Discovering Complexity: Decomposition and Localization as Strategies in Scientific Research. Princeton, NJ: Princeton University Press.

Bickle, J. (2003). Philosophy and Neuroscience: A Ruthlessly Reductive Account. Dordrecht: Kluwer.

Churchland, P. S. (1986). Neurophilosophy: Toward a Unified Theory of Mind-brain. Cambridge, MA: MIT Press.

Craver, C. (2007). Explaining the Brain: What a Science of the Mind-brain Could Be. New York: Oxford University Press.

Craver, C., \& Bechtel, W. (2007). Top-down Causation without Top-down Causes. Biology and Philosophy, 22, pp.547-563.

Fodor, J. A. (1974). Special Sciences (or: the Disunity of Science as a Working Hypothesis). Synthese, 28, pp.97-115.

Glennan, S. (2002). Rethinking Mechanistic Explanation. Philosophy of Science, 69, S342-S353.

Goldbeter, A. (1995). A Model for Circadian Oscillations in the Drosophila Period Protein (PER). Proceedings of the Royal Society of London. B: Biological Sciences, 261(1362), pp.319-324.

Halberg, F. (1959). Physiologic 24-hour Periodicity: General and Procedural Considerations with Reference to the Adrenal Cycle. Zeitschrift für Vitamin-, Hormonund Fermentforschung, 10, pp.225-296. 
Hardin, P.E., Hall, J.C., \& Rosbash, M. (1990). Feedback of the Drosophila period Gene Product on Circadian Cycling of its Messenger RNA Levels. Nature, 343(6258), pp.536-540.

Helfrich-Förster, C. (1996). Drosophila Rhythms: From Brain to Behavior. Seminars in Cell \& Developmental Biology, 7(6), pp.791-802.

Hempel, C.G. (1965). Aspects of Scientific Explanation. In C.G. Hempel (Ed.), Aspects of Scientific Explanation and Other Essays in the Philosophy of Science (pp.331-496). New York: Macmillan.

Inouye, S.-I.T., \& Kawamura, H. (1979). Persistence of Circadian Rhythmicity in a Mammalian Hypothalamic "Island" Containing the Suprachiasmatic Nucleus. Proceedings of the National Academy of Sciences (USA), 76, pp.5962-5966.

Konopka, R.J., \& Benzer, S. (1971). Clock Mutants of Drosophila melanogaster. Proceedings of the National Academy of Sciences (USA), 89, pp.2112-2116.

Leloup, J.-C., \& Goldbeter, A. (1998). A Model for Circadian Rhythms in Drosophila Incorporating the Formation of a Complex between the PER and TIM Proteins. Journal of Biological Rhythms, 13(1), pp.70-87.

Machamer, P., Darden, L., \& Craver, C. (2000). Thinking about Mechanisms. Philosophy of Science, 67, pp.1-25.

Nagel, E. (1961). The Structure of Science. New York: Harcourt, Brace.

Panda, S., \& Hogenesch, J.B. (2004). It's all in the Timing: Many Clocks, many Outputs. Journal of Biological Rhythms, 19(5), pp.374-387.

Roenneberg, T., Daan, S., \& Merrow, M. (2003). The Art of Entrainment. Journal of Biological Rhythms, 18(3), pp.183-194.

Welsh, D.K., Logothetis, D.E., Meister, M., \& Reppert, S.M. (1995). Individual Neurons Dissociated from Rat Suprachiasmatic Nucleus Express Independently Phased Circadian Firing Rhythms. Neuron, 14(4), pp.697-706.

Welsh, D.K., Yoo, S.-H., Liu, A.C., Takahashi, J.S., \& Kay, S.A. (2004). Bioluminescence Imaging of Individual Fibroblasts Reveals Persistent, Independently Phased Circadian Rhythms of Clock Gene Expression. Current Biology, 14(24), pp.2289-2295. 